\title{
Acute-stress-induced change in salience network coupling prospectively predicts post-trauma symptom development
}

Wei Zhang $\left(^{1,2}{ }^{凶}\right.$, Reinoud Kaldewaij ${ }^{1,2}$, Mahur M. Hashemi ${ }^{1,2}$, Saskia B. J. Koch (DD $^{1,2}$, Annika Smit (D) ${ }^{3,4}$, Vanessa A. van Ast (D) ${ }^{5}$, Christian F. Beckmann ${ }^{1,6,7}$, Floris Klumpers ${ }^{1,2,8}$ and Karin Roelofs (D) $^{1,2,8}$

(c) The Author(s) 2022

Substantial individual differences exist in how acute stress affects large-scale neurocognitive networks, including salience (SN), default mode (DMN), and central executive networks (CEN). Changes in the connectivity strength of these networks upon acute stress may predict vulnerability to long-term stress effects, which can only be tested in prospective longitudinal studies. Using such longitudinal design, we investigated whether the magnitude of acute-stress-induced functional connectivity changes (delta-FC) predicts the development of post-traumatic stress-disorder (PTSD) symptoms in a relatively resilient group of young police students that are known to be at high risk for trauma exposure. Using resting-state fMRI, we measured acute-stress-induced delta-FC in 190 police recruits before (baseline) and after trauma exposure during repeated emergency-aid services (16-month follow-up). Delta-FC was then linked to the changes in perceived stress levels (PSS) and post-traumatic stress symptoms (PCL and CAPS). Weakened connectivity between the SN and DMN core regions upon acute-stress induction at baseline predicted longitudinal increases in perceived-stress level but not of post-traumatic stress symptoms, whereas increased coupling between the overall SN and anterior cerebellum was observed in participants with higher clinician-rated PTSD symptoms, particularly intrusion levels. All the effects remained significant when controlling for trauma-exposure levels and cortisol-stress reactivity. Neither hormonal nor subjective measures exerted similar predictive or acquired effects. The reconfiguration of large-scale neural networks upon acute-stress induction is relevant for assessing and detecting risk and resilience factors for PTSD. This study highlights the SN connectivitychanges as a potential marker for trauma-related symptom development, which is sensitive even in a relatively resilient sample.

Translational Psychiatry (2022)12:63; https://doi.org/10.1038/s41398-022-01798-0

\section{INTRODUCTION}

Exposure to severely stressful events can lead to a wide range of mental health problems, including post-traumatic stress symptoms [1]. As most individuals with traumatic experiences do not develop significant symptoms in the long term [2], an important question is what psychological and biological processes constitute resilience to trauma [3]. So far, the vast majority of research studying the neural mechanisms underlying stress-related psychopathology has adopted cross-sectional designs. These studies have identified a number of critical brain regions that may be involved in resilience, but could also potentially reflect the symptoms that have already arisen [4]. A smaller number of studies, with generally limited sample sizes, has assessed which brain response prospectively predicts later trauma-symptom development by taking advantage of longitudinal designs with fMRI scans acquired before trauma exposure [5]. Interestingly, converging evidence from these studies suggests hyperactive amygdala as a predisposing factor for PTSD [4-6]. However, these studies have largely used task-based fMRI that is tailored to investigate targeted neural regions of interest, and thus generally leave open the question regarding the involvement of larger-scale neural networks, beyond the regional focus [5].

Acute stress has been shown to induce a reallocation of resources from three large-scale neural networks: the salience $(\mathrm{SN})$, default mode (DMN), and central executive network (CEN) $[7,8]$. This neural network reconfiguration has been speculated to prioritize resources to facilitate processing of challenging situations in health [7-9]. However, frequent and chronic exposure to stressors likely leads to unfavorable consequences, such as stressrelated disorders that have been associated with altered connectivity of SN and DMN [4, 10, 11]. To date, it remains unclear whether the magnitude of brain-network reorganization upon short-term challenges is predictive of one's vulnerability to or resilience against the negative consequences of long-term trauma exposure. Here, using a well-powered prospective longitudinal design, we tested this question in 190 Dutch police recruits who experienced a variety of potentially traumatic events in the line of duty.

Abnormal hyperconnectivity between the core regions of the SN (e.g., amygdala-insula and amygdala-dACC), and

\footnotetext{
'Donders Institute, Centre for Cognitive Neuroimaging, Radboud University, Nijmegen, The Netherlands. ${ }^{2}$ Behavioural Science Institute, Radboud University, Nijmegen, The Netherlands. ${ }^{3}$ Knowledge and Research Department, Police Academy of The Netherlands, Apeldoorn, The Netherlands. ${ }^{4}$ University of Humanistic studies, Utrecht, The Netherlands. ${ }^{5}$ Department of Clinical Psychology, University of Amsterdam, Amsterdam, The Netherlands. ${ }^{6}$ Department of Cognitive Neuroscience, Radboud University Medical Centre, Nijmegen, The Netherlands. ${ }^{7}$ Oxford Centre for Functional Magnetic Resonance Imaging of the Brain (FMRIB), University of Oxford, Oxford, UK. ${ }^{8}$ These authors contributed equally: Floris Klumpers, Karin Roelofs. ${ }^{\circledR}$ email: wzzang@me.com
}

Received: 3 July 2021 Revised: 6 January 2022 Accepted: 12 January 2022

Published online: 16 February 2022 
hypo-connectivity between the core regions of the DMN (e.g., vmPFC-PCC), as well as between core regions of these two networks (e.g., amygdala-vmPFC), were observed in PTSD patients in contrast to healthy controls [12-15]. In addition to SN and DMN, previous studies also reported decreased CEN connectivity upon acute-stress induction in a task condition, and the functional disruptions of the CEN in relation to PTSD [10, 16-21]. It therefore has been proposed that hypoactive DMN and CEN that are overwhelmed by a hyperactive and strongly connected SN may better characterize PTSD [15]. Yet, due to the nature of crosssectional designs with post-trauma assessments, these studies cannot inform whether the observed abnormalities existed prior to trauma exposure or were acquired along symptom development. The scarce pioneering longitudinal studies with small sample sizes (e.g., N between 15 and 50) [22-24] were likely statistically underpowered according to recent guidelines $[25,26]$ and therefore require replications with large sample sizes. Besides, although these studies provided hints for a linkage between the altered functions of core regions of the SN, DMN, CEN, and stressrelated disorders, only very few investigations directly deployed network-level approaches.

Since aberrant functional organizations of DMN, SN, and CEN have been proposed to underlie a wide range of psychopathologies, including stress-related ones such as depression, anxiety, and PTSD [10], it is critical to empirically test whether networklevel connectivity profiles in fact can prospectively impact subsequent symptom development after real-life trauma exposure and to elucidate whether these neural correlates can serve as potential risk or resilience factors. Our recent study examining network-connectivity changes in response to acute-stress induction demonstrated that connectivity changes of the SN (including dorsal anterior cingulate cortex, anterior insula, and amygdala) and DMN (including posterior cingulate cortex, precuneus and ventromedial prefrontal cortex) were correlated with cortisol increases after stress induction, respectively [8]. Given the implication of cortisol in inhibiting sympathetic stress responses and regaining physiological homeostasis following acute stressors $[27,28]$, the results from this previous work suggested an adaptive reorganization of brain functional networks in the face of challenges. With these findings, as well as the evidence showing the involvement of the SN, DMN, and CEN in stress-related symptoms [11, 15], we asked whether these short-term adaptive responses at the neural-network level could predict long-term consequences as a function of trauma exposure. Although stressinduced dynamical changes may be key to the identification of stress-resilience and vulnerability factors [3], such changes within and between these large-scale networks have not prospectively been tested in relation to long-term consequences after trauma exposure.

Using a longitudinal design, the current study aimed to elucidate whether the magnitude of acute-stress-induced network reconfiguration could predict stress-related symptom development after trauma exposure. Specifically, we used acute-stressinduced connectivity changes (i.e., delta-FC) of the SN, DMN, and CEN at baseline (i.e., Wave-1 assessment) to predict the perceived stress and PTSD symptom levels in police recruits after continuous exposure to police-operation-related trauma in the training period. We further investigated acquired abnormalities in network responses to stress induction after trauma exposure, using neuroimaging data collected at the follow-up assessment (i.e., on average 16 months after the baseline assessment).

Based on the literature, we expected to observe predictive effects of acute -stress-induced delta-FC in SN and DMN on trauma-related symptom development. More specifically, the adaptive responses to acute stress that were indicated by increasing SN and decreasing DMN connectivity at the baseline assessment, as shown in our previous study [8], were expected to be associated with lower levels of stress and PTSD symptoms after trauma exposure. For acquired effects, individuals with higher post-trauma stress levels were expected to show intensified SN connectivity and reduced DMN connectivity after stress induction as suggested in the PTSD literature [12-15]. CEN connectivity changes after acute stress induction were not associated with individual cortisol stress responses in our previous work [8]. However, given prior observations of aberrant CEN connectivity in stress-related psychopathologies [10,16-21], we further explored whether acute-stress-induced delta-FC of CEN could predict longterm post-trauma stress levels.

\section{MATERIALS AND METHODS \\ Participants}

An initial sample of 321 police recruits from Dutch Police Academy participated in the current study in accordance with the principles of the Declaration of Helsinki and with the approval from the Independent Review Board Nijmegen (IRBN), the Netherlands. All participants gave their written informed consent before the study upon their first lab visit (Wave1). Exclusion criteria included any current psychiatric or neurological disorder, history of, or current endocrine or neurological treatment, current use of psychotropic medication, and current drug or alcohol abuse (full details in protocol article [29]). A group of participants $(N=86)$ reported that their core trauma, the trauma most central to their symptoms, had occurred already before our baseline assessment (Wave-1). As we cannot disentangle predisposed and acquired factors for these participants due to the lack of data before Wave- 1 assessment, data from these participants were excluded. Further exclusion included data from the participant whose baseline PCL-5 scores met PTSD diagnosis cutoff (i.e., above 33) or who exhibited excessive motion artifacts (i.e., top $5 \%$ of participants showing the largest head motion effects from each fMRI session with the mean framewise displacement up to $0.39 \mathrm{~mm}$ ) [8], resulting in a final sample of 190 participants (mean age $=23.88$ ) that had complete data from all measurements (see Fig. 1 for detailed sample selection and Table 1 for demographic information).

\section{Procedure}

The baseline assessment (i.e., Wave-1) took place in parallel with the early police curriculum of mostly in-class theoretical trainings. In this assessment, participants filled out questionnaires measuring their baseline levels of perceived stress (using Perceived Stress Scale, PSS) and stress-related symptoms (using PTSD Checklist for DSM-5, PCL-5), prior to the participation in all other experimental tasks. Acute stress induction was conducted in the late afternoon (i.e., between 4 and $7 \mathrm{pm}$ ) to ensure stable salivary cortisol levels, which consisted of a SECPT (Socially Evaluated Cold Pressure Task) and MA (mental arithmetic) task (see details in the Supplemental Materials and Methods) $[8,30,31]$. Hormonal and subjective stress responses were assessed multiple times throughout the experiment (i.e., approximately at $-10,0,+10,+20$, and +30 minutes with respect to the experiment onset), and two sessions of resting-state fMRI (rs-fMRI) data with identical acquisition length of 500 scans (367.5 s) were acquired immediately before and after stress induction to assess stress-induced functional connectivity changes (Fig. 2). After an average of 16 months $(S D=1.9)$, participants were tested in the follow-up assessment (i.e., Wave2 ), when identical measurements were repeated to investigate consequences of exposure to trauma-like events. In the Wave- 2 assessment, the Police Life Event Scale (PLES) [32] was additionally used to index the amount of trauma exposure. Participants also participated in a telephone interview comprising the Clinician-Administered PTSD Scale for DSM-5 (CAPS, see full details about all measurements in the protocol article [29]).

\section{Data acquisition and analysis}

Imaging-data acquisition. Acquisition of the resting-state fMRI data occurred before and after the stress-induction experiment in both Wave-1 and Wave-2 assessments, where participants were instructed to lie still and watch a small white cross at the screen center. Imaging data were acquired using a 3-T Siemens Magnetom Prisma ${ }^{\text {fit }}$ MRI scanner (Erlangen, Germany) with a 32-channel head coil. A multi-band T2*-weighted EPI sequence with acceleration factor 8 (MB8), optimized from the recommended imaging protocols for the Human Connectome Project, was used to acquire a total number of 500 volumes of BOLD-fMRI images (TR $=735 \mathrm{~ms}$, TE $=39 \mathrm{~ms}$, flip angle $=52^{\circ}$, voxel size $=2.4 \times 2.4 \times 2.4 \mathrm{~mm}^{3}$, slice gap $=0 \mathrm{~mm}$, and 


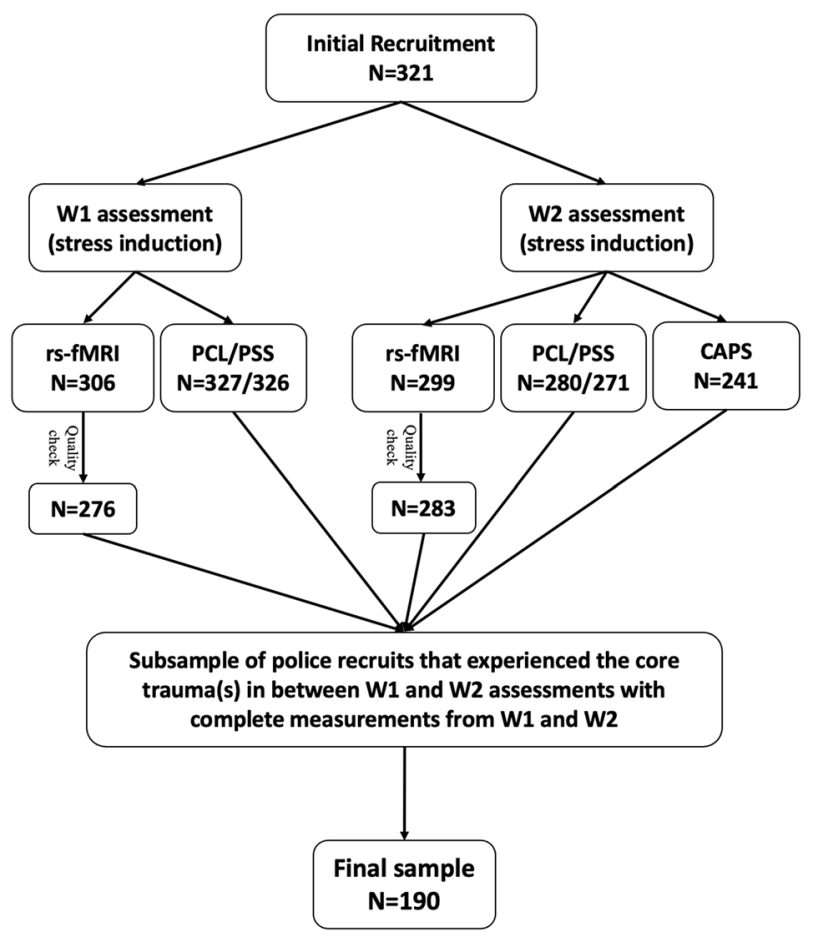

Fig. 1. Flow Chart of Study Participants. A total number of 321 police recruits participated in the current study. Data from a subsample were acquired for resting-state functional MRI (rs-fMRI) and stress-level-related questionnaires (i.e., PTSD Checklist, PCL; Perceived Stress Scale, PSS) or interviews (i.e., Clinician-Administered PTSD Scale, CAPS), at both Wave- 1 and Wave-2 assessments. Following data quality check and the screening of core trauma experiences (i.e., occurrence in-between two assessments), data from a final sample of 190 participants were used for further analyses.

Table 1. Sample demographics.

\begin{tabular}{|llll} 
Sex & Sample size & Age $(\mathbf{m} / \mathbf{s d})$ & $\begin{array}{l}\text { Experienced trauma } \\
\text { types }(\mathbf{m} / \mathbf{s d})\end{array}$ \\
\hline Female & 48 & $23.84 / 4.67$ & $5.04 / 3.14$ \\
\hline Male & 142 & $24.0 / 5.69$ & $4.8 / 3.5$ \\
\hline
\end{tabular}

FOV $=210 \mathrm{~mm})$. High-resolution structural images $\left(1 \times 1 \times 1 \mathrm{~mm}^{3}\right)$ were also acquired, using a T1-weighted MP-RAGE sequence $(T R=2300 \mathrm{~ms}$, TE $=$ $3.03 \mathrm{~ms}$, flip angle $=8^{\circ}$, and FOV $=256 \times 256 \times 192 \mathrm{~mm}^{3}$ ).

Assessment of stress-related measures. Perceived stress-level and PTSDsymptom levels were measured at both the baseline (Wave-1) and followup (Wave-2), prior to the implementation of the acute-stress-induction experiment (see "Procedure"). Additionally, CAPS interviews were conducted at Wave-2 assessment. While sum scores of CAPs were used to indicate post-traumatic symptom levels, the change scores of PSS (i.e., Wave-1 score subtracted from Wave- 2 score, delta-PSS) and PCL (delta$\mathrm{PCL}$ ) were calculated to indicate the development of post-traumatic stress levels.

Assessment of stress-induced hormonal and behavioral measures. To index acute endocrine- and subjective-stress responses, salivary samples and self-reported ratings of negative affect were measured throughout the stress-induction experiment in Wave- 1 and Wave- 2 assessments. Following our previous practice [8], increases in salivary cortisol and negativeaffect ratings were calculated for each participant to index the magnitude of acute-stress responses. Specifically, cortisol increase was defined as the cortisol level 20 minutes after stress-induction onset (i.e., at time +20 min. when responses peaked) subtracting baseline level immediately before

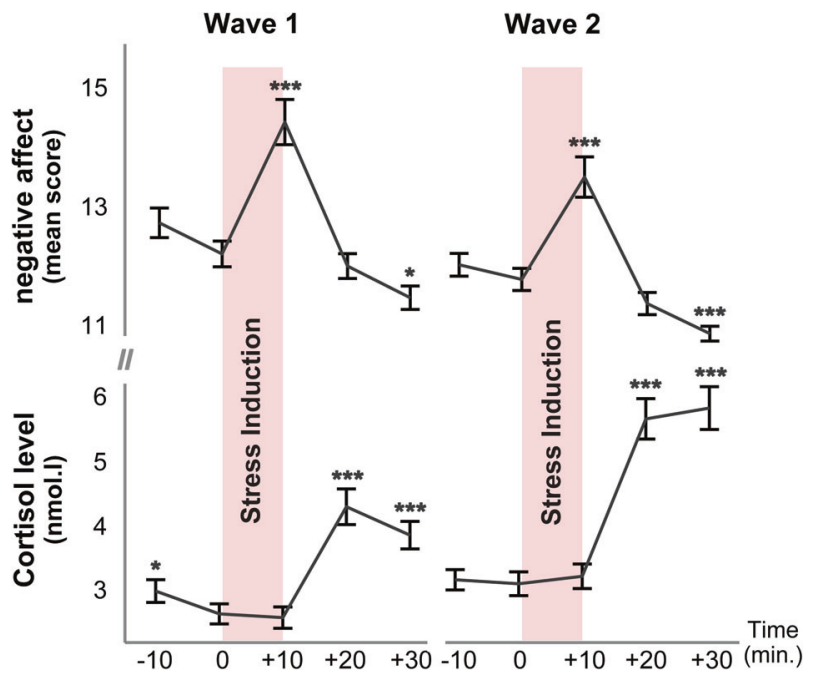

Fig. 2 Hormonal and behavioral responses to acute-stress induction. Acute-stress induction has led to comparable response patterns in hormonal and subjective measures in Wave-1 and Wave2 assessments. Negative affect peaked immediately after the onset of stress induction and declined thereafter, until eventually below the prestress baseline level. Cortisol showed the expected downward trend observed after the morning peak, which was followed by a peak at 20 minutes after the onset of stress induction and remained high. Although overall cortisol increases (i.e., the difference between +20 and 0 minutes) were larger in Wave- 2 than in Wave-1 assessments $(p<0.05)$, no differences were observed in negative-affect responses (i.e., the difference between +10 and 0 minutes). Error bars represent SEM (standard error of measurement) and asterisks indicate significant differences relative to the pre-stress baseline level at time 0 min. ${ }^{* *} p<0.0001$; ${ }^{*} p<0.05$.

stress induction (i.e., at time $0 \mathrm{~min}$.). Negative-affect increase was calculated as the difference in ratings between the baseline (time $0 \mathrm{~min}$.) and 10 minutes after the onset of stress induction (time $+10 \mathrm{~min}$. Fig. 2).

\section{fMRI preprocessing and analysis}

Preprocessing: Preprocessing of rs-fMRI data included motion correction, $5 \mathrm{~mm}$ spatial smoothing, ICA-AROMA-based denoising [33], and high-pass filtering with a cutoff of 100 seconds. Mean signal intensity of white matter and cerebrospinal fluid, as well as head-motion parameters were regressed out to minimize psychophysiological confounds and motion artifacts $[34,35]$. The resulting residual images were subsequently registered to the $\mathrm{MNI}$ atlas and used for statistical analyses. Detailed preprocessing can be found in the Supplemental Materials and Methods.

Identifying delta-FC of resting-state networks. Resting-state networks (RSNs) of interest (i.e., SN, DMN, and CEN) were identified from the components of a group-level independent-component analysis (ICA) that showed the highest spatial correlation with preselected functional ROIs (i.e., anterior SN, left CEN, right CEN, and ventral DMN) from the Stanford FIND atlas [8]. Changes in acute-stress-induced functional connectivity (i.e., delta-FC) of these RSNs were defined as the connectivity difference between scans pre- and post-stress induction in each of three RSNs (i.e., $\mathrm{SN}, \mathrm{DMN}$, and CEN). Importantly, we defined the delta-FC at two levels: the local level refers to the delta-FC within each individual RSN, whereas the global level indicates the delta-FC of each RSN with the brain regions also outside the predefined networks. Coefficients of both local and global delta-FC were included in the analyses.

Testing predictive and acquired effects. To indicate the magnitude of changes in functional connectivity as a function of acute-stress induction, coefficients of delta-FC were extracted using the predefined local and global networks. Both local and global delta-FC coefficients from Wave-1 assessment were used to predict stress-related symptom development (i.e., changes in symptoms between two assessments). In contrast, to assess 
potential acquired changes in network-connectivity strength consequential to trauma, we calculated changes of these delta-FC coefficients over time (from Wave-1 to Wave-2) and looked at the associations with symptom development.

To obtain the local-level delta-FC coefficients, we used the RSN maps derived from group-level ICA with a threshold of $Z>3$ to reduce noise while retaining the overall spatial patterns of each network. The changes in the beta-values within the resulting mask were then used to index the network-connectivity changes after stress induction. For the more global-level delta-FC coefficients, we calculated the differential RSNs per network at the individual level (i.e., derived from dual-regression analysis) before and after stress induction (i.e., subtraction between two scans), thresholded at $p<0.0167$ (i.e., accounting for three RSNs), and averaged the resulting maps across participants. This essentially created a group-level map of regions showing consistent changes in connectivity with the network of interest after stress induction. Changes in the averaged beta-values across all these regions were taken as a summary measure of stress-related global connectivity changes for that network (also see Supplemental Materials and Methods) [8]. This two-level network approach allowed us to examine each individual RSN upon the perturbation by acute-stress induction for the internal communications among nodes (i.e., local-level delta-FC), as well as for their interplays with all brain areas that showed a connectivity change with the network (i.e., global-level delta-FC).

Additionally, as cortisol-stress reactivity was previously found predictive of subsequent PTSD-symptom development in a limited number of studies [36, 37], we tested whether this could be replicated by using acute-stress-induced cortisol increases at baseline to predict stressrelated symptomatology in our study. We further examined the changes in cortisol increase and negative affect upon acute-stress induction as a function of trauma exposure to explore whether symptom changes are accompanied by hormonal and behavioral changes (i.e., acquired effects).

Delta-PSS, delta-PCL, and CAPS sum scores were calculated to indicate each individual's post-traumatic stress levels. These change scores were used as the outcome measures in our analyses for predictive and acquired effects of symptom development. We further explored the development of specific symptom clusters, using the sum scores of each subcluster in delta-PCL and CAPS measures (Table S1).

\section{Statistical analyses}

Spearman rank correlation was used for all correlation analyses to mitigate the influences from extreme values and reduce the chance of false positives. Concerning the results for our a priori hypotheses (i.e., regarding the delta-FC of SN and DMN), FDR corrections were applied to account for the number of analyses involving three outcome measurements (i.e., deltaPCL, delta-PSS, and CAPS scores). For more exploratory analyses concerning the delta-FC of CEN for which we had no a priori hypotheses, more stringent FDR corrections were considered to account for two levels of network connectivity (i.e., local and more global levels) and three outcome measurements. Follow-up tests on subcluster symptom scores were carried out only if significant predictive or acquired effects were observed. Concerning these analyses, FDR corrections were conducted to account for the number of analyses involving all four subcluster symptom scores, for the local- and more global-level connectivity, separately. In case of significant results concerning delta-PCL or delta-PSS (either the overall changing score or the subcluster score), semipartial Spearman correlation was further conducted to control for the baseline PCL or PSS level from Wave- 1 assessment. Finally, we used a generalized additive model (GAM) to explore whether the predictive effects of hypothesized neural measures remain significant when accounting for influences of stress reactivity at hormonal and behavioral levels from baseline assessment, and the impact of trauma exposure (i.e., indicated by the number of experienced trauma types). Similar analyses were conducted for acquired effects to account for changes in cortisol and negative-affect stress responses between two assessments. Unlike multiple linear regression that estimates a single parameter for each predictor, GAM finds unspecified (nonparametric) functions that relate the predicted $Y$ (dependent variable) values to the predictor values, and thus allows nonparametric fit [38, 39].

Apart from the coefficient extraction that was carried out using FSL [40], all other statistical analyses were conducted using $R$ version 3.6.1 [41], with pcor function from RVAideMemoire package [42] and gam function from mgcv package [43] specifically for running semipartial correlation and GAM analyses, respectively.

\section{RESULTS}

\section{Acute-stress responses}

Successful acute-stress induction was observed in both baseline (Wave-1) and follow-up (Wave-2) assessments. Specifically, increases in salivary cortisol and the reported negative affect were observed in Wave-1 following stress induction, as reflected in the main effects of sampling time $\left(\mathrm{F}_{\text {cortisol }}(4677.36)=76.82, p<\right.$ $\left.0.0001 ; \quad F_{\text {affect }}(4719.87)=51.50, p<0.005\right)$. Similar significant effects were observed in Wave-2 $\left(F_{\text {cortisol }}(4644.18)=123.4, F_{\text {affect }}\right.$ $\left.(4675.06)=51.03, p^{\prime} s<0.0001\right)$. In short, our experimental manipulation successfully induced acute stress, indicated by increases in cortisol and negative effect for both waves (see Fig. 2).

\section{Traumatic experiences and post-traumatic stress measures}

In-between our two waves of data collection, the police recruits on average experienced 4.92 different types of potentially traumatic events $(S D=3.48)$ with a range between 0 and 17 . The most frequently experienced trauma were encountering suicide (including attempt, 31.4\%), severe (traffic) accidents (23.6\%), and physical assault (17.8\%, Fig. S1).

From Wave1 to Wave-2, average stress-symptom levels slightly increased with no statistically significant changes at the group level in either perceived stress (PSS: $t(182)=1.48, p=0.14$ ) or overall PTSD-symptom levels (PCL: $t(189)=0.77, p=0.44)$. Nevertheless, closer inspection revealed large variance in individualsymptom trajectories (Fig. 3; Table S2), together with a significant increase in intrusion-symptom level $(t(189)=2.22, p<0.05$; other symptom clusters $p>0.05)$. At Wave-2, the average clinical interview (CAPS) score of overall PTSD symptoms was 1.79 ( $S D=4.01$; sum-score range: $0-27$, with three participants having developed full-blown PTSD according to DSM-5 criteria (Fig. S2). Although the group-level CAPS score was relatively low, a substantial proportion of police students (about $80 \%$ ) exhibited clinically relevant increases in symptom levels (i.e., reported at least one symptom in each cluster in Wave-2 PCL). We therefore proceeded to test whether the large variation in PTSD-symptom trajectories could be explained by the stress-related neuralnetwork-connectivity changes.

\section{Predictive effects of baseline acute-stress responses for subsequent trauma-symptom development}

In testing our a prior hypotheses concerning the SN and DMN, a decreased coupling between $\mathrm{SN}$ and DMN core regions (i.e., posterior cingulate cortex/precuneus) following stress induction at baseline was found predictive of larger increases in perceived stress level after trauma exposure ( $r$ o $=-0.19, p=0.0094$, Fig. 4). This effect remained significant after FDR correction for multiple comparisons $\left(p_{\mathrm{fdr}}<0.0167\right)$ and when baseline level PSS was controlled for ( $r h o=-0.19, p=0.0039$ ). This predictive effect of SN-DMN coupling remained as the only significant predictor in our follow-up analysis using generalized additive model (GAM) when influences of baseline cortisol and subjective affect, as well as trauma exposure amount were considered $(F=7.20, p=0.008)$. Against our hypotheses, we observed no predictive effects of delta-FC in SN on self-reported PTSD symptoms (delta-PCL) or clinician-rated PTSD levels (CAPS scores), nor with respect to hypothesized DMN-connectivity changes after stress induction (all uncorrected $p^{\prime} s>0.08$ ).

Subsequent exploratory analyses for CEN revealed that higher clinician-rated PTSD symptoms (i.e., CAPS total score) were predicted by increased delta-FC, both at the local (i.e., delta-FC within the $C E N$; rho $=0.21, p=0.0031$ ) and more global levels (i.e., delta-FC of CEN with brain regions also outside the network; Rs = $0.19, p=0.0089$ ). After FDR corrections, only the effect of local CEN delta-FC remained significant $\left(p_{\mathrm{fdr}}<0.019\right)$. Follow-up tests on the subcluster symptoms revealed that delta-FC within CEN predicted levels of alteration in mood and cognition ( $r$ ho $=0.19$, $p=0.0085$ ), as well as hyper-arousal symptoms ( $\mathrm{rho}=0.25$, 

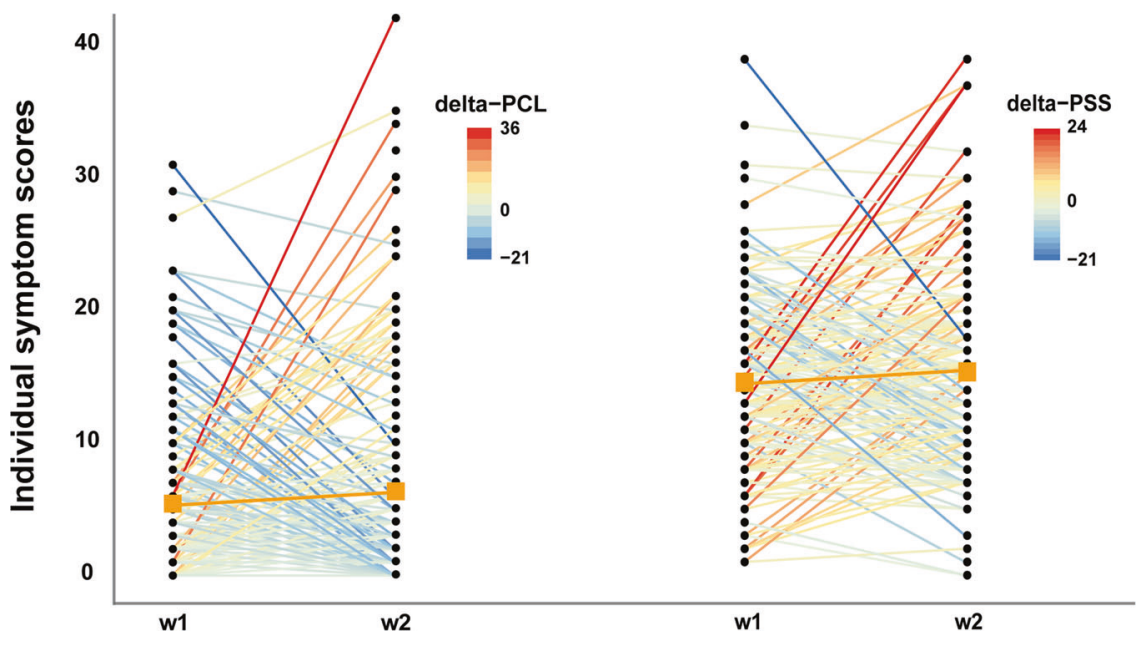

Fig. 3. Stress-related Symptom Changes. Development of PTSD-symptom levels (assessed by PCL-5) and perceived stress levels (assessed by PSS) from Wave-1 (w1) to Wave-2 (w2) were illustrated. Large individual differences were observed for change scores in PCL (i.e., delta-PCL, left panel, range: $-21-+36$ ), and in PSS (i.e., delta-PSS, right panel, range $-21-+24)$. Each line represents the individual change in stress symptoms from w1 to w2. Group means for both PCL and PSS from each wave assessment are illustrated using the orange squares.

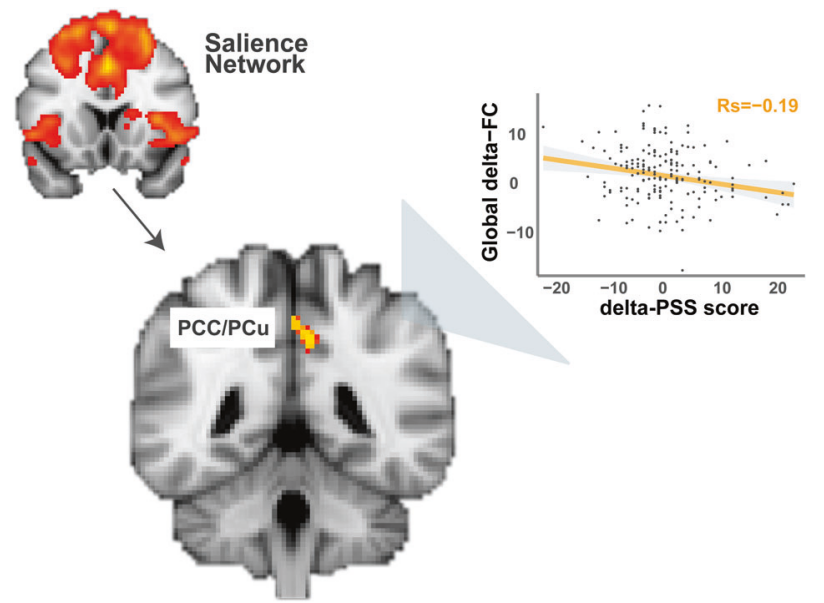

Fig. 4 Predictive Effect of SN Connectivity on Post-trauma Stress Levels. Decreases in the coupling between the overall SN and hub regions of the DMN (i.e., posterior cingulate cortex (PCC)/precuneus (PCu)), and postcentral gyrus and intracalcarine cortex as a function of acute-stress induction predicted the higher levels of perceived stress after trauma exposure. The arrow indicates functional connectivity changes between the overall salience network (i.e., coefficients extracted using the depicted network mask thresholded at $\mathrm{Z}>3$ ) and clusters in PCC/PCu, visual cortex, and somatosensory cortex.

$p=0.00058)$. These effects also remained significant after multiple-comparison correction ( $p_{\mathrm{fdr}}<0.035$; Fig. S3).

In comparison to these baseline neural responses to acutestress induction, we did not find cortisol reactivity, nor negative effect, predictive of stress-related symptomology development (all $\left.p^{\prime} s>0.05\right)$.

\section{Acquired effects after trauma exposure}

Changes in acute-stress responses from Wave- 1 to Wave- 2 at hormonal, behavioral and neural levels were linked to increases in symptomology to test for acquired abnormalities. Although an increase was observed at the group level in cortisol stress responses from Wave- 1 to Wave-2 assessments $(t(150)=3.52$, $p<0.001$ ), we did not find any associations between this increased cortisol-stress response and symptom level changes (all $p^{\prime} s>0.05$ ). No difference in negative affect levels upon acute stress induction was found between Wave-1 and Wave-2 assessments, nor did we observe any significant associations between negative affect and symptom changes (all $p^{\prime} s>0.05$ ). Furthermore, we did not observe significant associations between connectivity changes of DMN or CEN and symptom changes (all $\left.p^{\prime} s>0.05\right)$. However, we did observe an increased coupling between the overall SN and anterior cerebellum (i.e., increased delta-FC as a function of acute stress induction) from Wave- 1 to Wave-2 that was associated with higher PTSD symptom levels (i.e., CAPS total score, rho $=-0.18, p=0.019$ ). Yet, this effect just missed significance when correcting for multiple comparisons $\left(p_{\mathrm{FDR}}=0.057\right)$. Follow-up tests examining sub-cluster symptoms suggested that this effect might have been driven by intrusion symptom (rho $=-0.22, p=0.0038$; other symptoms $p>0.05$ ), with the participants showing higher intrusion-symptom levels also exhibiting larger neural coupling in response to acute stress induction (Fig. 5). The effect for subcluster symptom also remained significant after FDR correction $\left(p_{\mathrm{FDR}}=0.015\right)$, and after accounting for the influence of changes in cortisol and negativeaffect stress responses between two assessments $(F=6.05, p=$ $0.015)$. No significant effects were observed for delta-PCL, nor for delta-PSS scores.

\section{DISCUSSION}

In this prospective longitudinal study, we investigated whether acute-stress-induced neural network changes could function as a risk factor of or a resilience factor against the development of PTSD symptoms. To this end, we tested the predictive effects of such changes on long-term stress-related symptomatology after exposure to real-life trauma in police recruits. Reduced global connectivity of the SN with DMN in response to baseline acute stressors predicted increased post-trauma stress levels 16 months later. A different pattern emerged for neural-network changes between assessments that followed symptom development and thus appear acquired rather than a pretrauma risk factor: individuals with higher levels of PTSD intrusions symptoms at follow-up showed increased coupling between the $\mathrm{SN}$ and anterior cerebellum after acute stress induction in the follow-up versus baseline assessment. Interestingly, both acquired and predictive neural effects in our study were found significant above and beyond the contribution of hormonal and subjective 


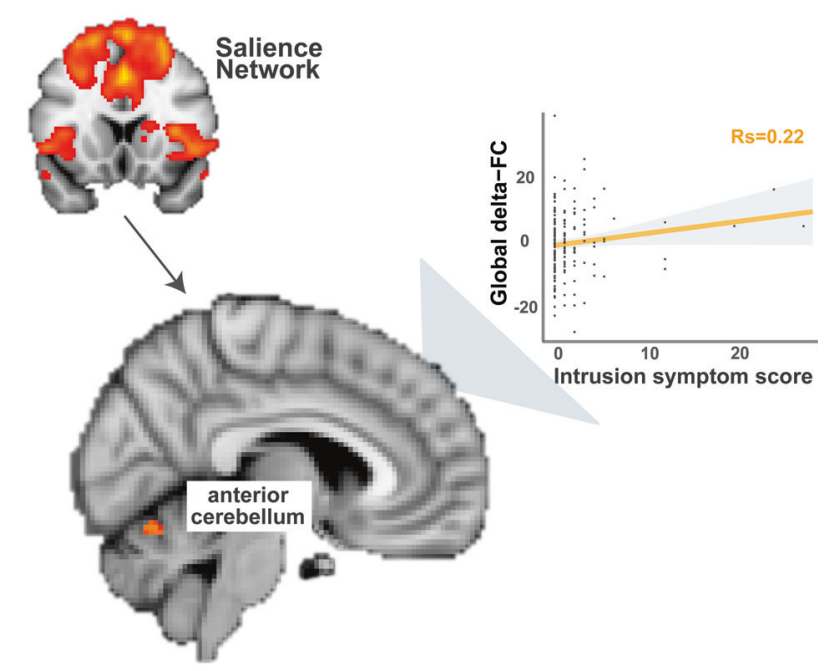

Fig. 5. Acquired Abnormality in SN Connectivity After Trauma Exposure. Increased coupling (stemming from reduced decoupling) between the overall SN and anterior cerebellum (as depicted in the brain image) from Wave1 to Wave2 in response to acute-stress induction was associated with higher levels of intrusion symptom, indicated by CAPS scores. The arrow demonstrates functional connectivity changes between the overall salience network (i.e., coefficients extracted using the depicted network mask thresholded at $Z>3$ ) and clusters in anterior cerebellum. To note, spearman rank correlation was conducted to minimize the influences from the extreme values.

stress measures. Together, these findings suggest that the $\mathrm{SN}$ connectivity patterns as a function of acute stress may serve as a potential risk factor to the development of stress-related symptoms upon trauma exposure.

To our knowledge, this is the first longitudinal study that has used a network-based approach to link stress-induced connectivity changing patterns of large-scale brain networks at baseline to the subsequent symptom development after trauma exposure. Our findings therefore provide new empirical evidence that the magnitude of large-scale network reconfiguration upon acutestress exposure is relevant for investigating resilience and risk factors for stress-related symptomatology.

In line with our predictions, acute-stress-induced decreases in overall SN connectivity with brain regions (i.e., PCC and precuneus) predicted higher perceived stress level after trauma exposure. Acute stress has been shown to immediately prompt SN engagement at the potential cost of neural resources that would otherwise have been allocated to other brain circuits $[7,8]$. This stress-induced reconfiguration of brain function is hypothesized to facilitate the coping with the challenging situations at hand by reallocating neural resource toward the SN for attention direction toward evolutionary-relevant stimuli and integration of top-down appraisal and bottom-up visceral and sensory information (see review by Uddin [44]). Insufficient SN involvement in response to acute stress therefore may signal suboptimal processing, such that its dynamic coordination with other brain networks becomes diminished and thus results in undesirable long-term consequences, such as the observed increases in stress levels after exposure to real-life trauma. Notably, hyperactivity of the amygdala and hypoactivity of the prefrontal cortex have consistently been implicated in stress-related psychopathology (see review by Fenster et al. [1]), including our previous work where diminished aPFC control over the amygdala during an approach and avoidance task predicted subsequent PTSD symptoms [6]. Yet, in the current study, more local-level SN connectivity with extracted coefficients also containing signals of bilateral amygdala did not show any predictive effects.
This discrepancy is likely due to different study designs: involvement of fronto-amygdala circuit is often seen in task conditions requiring repeated regulation of a series of task-related stimuli, suggesting complementary insight into PTSD biomarkers provided by different study designs.

Interestingly, SN reconfiguration upon acute-stress induction seemed to not only signal a risk factor for later symptom development, but also indicate acquired abnormalities that were associated with increasing symptom levels. Our finding of SN-cerebellum coupling in participants with relatively high PTSD symptoms is in line with a growing number of studies that has linked cerebellum to emotional processing and regulation, particularly to negative emotional memories [45-47], as well as to pathophysiology of PTSD [48-52].

Furthermore, our exploratory analyses for CEN found that acutestress-induced increases in connectivity of this network prior to trauma exposure could predict the elevated levels of overall posttrauma symptoms, and of specific negative mood/cognition and hyperarousal symptoms (Figure S3). These results suggest that the reconfiguration of CEN upon acute stressors may allow the tracking of symptom development after trauma exposure. Our findings are in line with the role of dorsal-lateral prefrontal cortex (dIPFC), a core region of the CEN in emotion regulation. They are also consistent with the evidence from stimulation studies that the modulation of dIPFC activity and connectivity could be beneficial in alleviating PTSD symptoms $[53,54]$.

Contrary to our prediction, however, we did not observe any associations between acute stress-induced delta-FC of DMN at baseline and long-term consequences of trauma exposure. However, the finding of decreased connectivity between SN and posterior DMN suggests that the regions in DMN may function distinctively in response to stressful events over time, hence, the mean coefficient indicating overall connectivity patterns of all regions within the network at baseline could not capture individual variability in longitudinal symptom development. Additionally, we did not find evidence in support of cortisol reactivity predicting PTSD-symptom development, which was reported in a few recent longitudinal studies [37, 55]. Discrepancy may arise from differences in sample characteristics (i.e., combat soldiers vs. police recruits), analytical approaches (i.e., subtyping vs. continuous modeling), and the timing of assessment with regard to trauma exposure (i.e., once a year for four years vs. twice with 16 months in-between) between the previous and our studies. Future investigations that study cortisol-stress response in relation to symptom development should consider these differences.

In an attempt to investigate the predictive factors for PTSDsymptom development and to disentangle predictive from acquired effects of the neural networks, we focused on a relatively healthy and resilient sample, whose baseline levels of depression, anxiety, and PTSD-symptom scores at Wave-1 assessment were significantly lower in comparison with a group of age- and sexmatched control participants (all $\mathrm{p}^{\prime} \mathrm{s}<0.05$ ). Notably, these observations were in line with the fact that all police students were prescreened for the enrollment based on their physical and psychological performance, and with the literature suggesting higher resilience in police than in civilians [56]. With limited variations in stress-related psychopathology in the current study sample, our study differs from most of the existing longitudinal (and cross-sectional) studies that have focused on clinical populations with maximized variances pertinent to the symptomology $[12,57]$. However, our sample here is not affected by the typical confounds that surround more severe psychopathology either (e.g., medication intake). Our approach therefore potentially allows more direct interpretations of the prediction findings and our resilient sample sheds important light on how stress resilience versus vulnerability might be instantiated in the brain. Further, an important advantage of this study was the leverage 
of a well-established acute-stress challenge in combination with a relatively large sample size.

In conclusion, the current study used connectivity changes of large-scale neural networks in response to an acute-stress challenge to predict subsequent stress-related symptoms after trauma exposure in police recruits. Whereas SN-DMN connectivity prospectively predicted the longitudinal changes in perceived stress level, increased SN-cerebellum connectivity was acquired in participants with higher PTSD-symptom levels. These findings suggest that acute-stress-induced SN-connectivity changes may serve as a potential marker of PTSD vulnerability.

\section{DATA AVAILABILITY}

The data and code supporting this study are stored in Donders Repository (https:// data.donders.ru.nl/) and are available from the corresponding author upon request.

\section{REFERENCES}

1. Fenster RJ, Lebois LAM, Ressler KJ, Suh J. Brain circuit dysfunction in posttraumatic stress disorder: from mouse to man. Nat Rev Neurosci 2018;19:535-51.

2. Galatzer-Levy IR, Huang SH, Bonanno GA. Trajectories of resilience and dysfunction following potential trauma: A review and statistical evaluation. Clin Psychol Rev. 2018. https://doi.org/10.1016/j.cpr.2018.05.008.

3. Kalisch R, Baker DG, Basten U, Boks MP, Bonanno GA, Brummelman E, et al. The resilience framework as a strategy to combat stress-related disorders. Nat Hum Behav 2017;1:784-90.

4. Admon R, Milad MR, Hendler T. A causal model of post-traumatic stress disorder: Disentangling predisposed from acquired neural abnormalities. Trends Cogn Sci. 2013;17:337-47.

5. Roeckner AR, Oliver KI, Lebois LAM, van Rooij SJH, Stevens JS. Neural contributors to trauma resilience: a review of longitudinal neuroimaging studies. Transl Psychiatry. 2021. https://doi.org/10.1038/s41398-021-01633-y.

6. Kaldewaij R, Koch SBJ, Hashemi MM, Zhang W, Klumpers F, Roelofs K. Anterior prefrontal brain activity during emotion control predicts resilience to posttraumatic stress symptoms. Nat Hum Behav. 2021. https://doi.org/10.1038/ s41562-021-01055-2.

7. Hermans EJ, van Marle HJF, Ossewaarde L, Henckens MJAG, Qin S, van Kesteren MTR, et al. Stress-related noradrenergic activity prompts large-scale neural network reconfiguration. Science. 2011;334:1151-3.

8. Zhang W, Hashemi MM, Kaldewaij R, Koch SBJ, Beckmann C, Klumpers F, et al. Acute stress alters the 'default' brain processing. Neuroimage. 2019;189:870-7.

9. Hermans EJ, Henckens MJAG, Joëls M, Fernández G. Dynamic adaptation of largescale brain networks in response to acute stressors. Trends Neurosci. 2014;37:304-14.

10. Menon V. Large-scale brain networks and psychopathology: A unifying triple network model. Trends Cogn Sci. 2011;15:483-506.

11. Koch SBJ, van Zuiden M, Nawijn L, Frijling JL, Veltman DJ, Olff M. Aberrant resting-state brain activity in posttraumatic stress disorder: $\mathrm{A}$ meta-analysis and systematic review. Depress Anxiety. 2016;33:592-605.

12. Bremner JD, Elzinga B, Schmahl C, Vermetten E. Structural and functional plasticity of the human brain in posttraumatic stress disorder. Prog Brain Res. 2007; 167:171-86.

13. Shin LM, Liberzon I. The neurocircuitry of fear, stress, and anxiety disorders. Neuropsychopharmacology 2010;35:169-91.

14. Wang T, Liu J, Zhang J, Zhan W, Li L, Wu M et al. Altered resting-state functional activity in posttraumatic stress disorder: A quantitative meta-analysis. Sci Rep 2016; 6. https://doi.org/10.1038/srep27131.

15. Akiki TJ, Averill CL, Abdallah CG. A network-based neurobiological model of PTSD: Evidence from structural and functional neuroimaging studies. Curr Psychiatry Rep. 2017; 19. https://doi.org/10.1007/s11920-017-0840-4.

16. Daniels JK, Mcfarlane AC, Bluhm RL, Moores KA, Richard Clark C, Shaw ME, et al. Switching between executive and default mode networks in posttraumatic stress disorder: Alterations in functional connectivity. J Psychiatry Neurosci. 2010;35:258-66.

17. Liu Y, Li L, Li B, Feng N, Li L, Zhang X et al. Decreased Triple Network Connectivity in Patients with Recent Onset Post-Traumatic Stress Disorder after a Single Prolonged Trauma Exposure. Sci Rep 2017; 7. https://doi.org/10.1038/s41598-01712964-6.

18. Miller GE, Chen E, Armstrong CC, Carroll AL, Ozturk S, Rydland KJ, et al. Functional connectivity in central executive network protects youth against cardiometabolic risks linked with neighborhood violence. Proc Natl Acad Sci USA. 2018;115:12063-8.
19. Aupperle RL, Melrose AJ, Stein MB, Paulus MP. Executive function and PTSD: disengaging from trauma. Neuropharmacology. 2012;62:686-94.

20. Polak AR, Witteveen $A B$, Reitsma JB, Olff $M$ The role of executive function in posttraumatic stress disorder: A systematic review. J. Affect. Disord. 2012. https:// doi.org/10.1016/j.jad.2012.01.001.

21. Lanius RA, Frewen PA, Tursich M, Jetly R, McKinnon MC. Restoring large-scale brain networks in ptsd and related disorders: A proposal for neuroscientificallyinformed treatment interventions. Eur J Psychotraumatol. 2015;6:1-12.

22. Admon R, Lubin G, Stern O, Rosenberg K, Sela L, Ben-Ami H, et al. Human vulnerability to stress depends on amygdala's predisposition and hippocampal plasticity. Proc Natl Acad Sci USA. 2009;106:14120-5.

23. McLaughlin KA, Sheridan MA, Lambert HK. Childhood adversity and neural development: Deprivation and threat as distinct dimensions of early experience. Neurosci Biobehav Rev. 2014;47:578-91.

24. Van Wingen GA, Geuze E, Vermetten E, Fernández G. Perceived threat predicts the neural sequelae of combat stress. Mol Psychiatry. 2011;16:664-71.

25. Elliott ML, Knodt AR, Ireland D, Morris ML, Poulton R, Ramrakha $S$ et al. What is the test-retest reliability of common task-functional MRI measures? New empirical evidence and a meta-analysis. Psychol Sci. 2020. https://doi.org/10.1177/ 0956797620916786

26. Button KS, loannidis JPA, Mokrysz C, Nosek BA, Flint J, Robinson ESJ, et al. Power failure: why small sample size undermines the reliability of neuroscience. Nat Rev Neurosci. 2013;14:365-76.

27. De Kloet ER, Joëls $M$, Holsboer F. Stress and the brain: From adaptation to disease. Nat Rev Neurosci 2005;6:463-75.

28. McEwen BS. Stress, adaptation, and disease: allostasis and allostatic load. Ann N Y Acad Sci. 1998;840:33-44.

29. Koch SBJ, Klumpers F, Zhang W, Hashemi MM, Kaldewaij R, van Ast VA, et al. The role of automatic defensive responses in the development of posttraumatic stress symptoms in police recruits: protocol of a prospective study. Eur J Psychotraumatol. 2017;8:1412226.

30. Schwabe L, Haddad L, Schachinger H. HPA axis activation by a socially evaluated cold-pressor test. Psychoneuroendocrinology. 2008;33:890-5.

31. Luo Y, Fernández G, Hermans E, Vogel S, Zhang Y, Li H et al. How acute stress may enhance subsequent memory for threat stimuli outside the focus of attention: DLPFC-amygdala decoupling. Neuroimage 2018. https://doi.org/10.1016/j. neuroimage.2018.01.010.

32. Carlier I, Gersons B. Development of a scale for traumatic incidents in police officers. Psychiatr Fenn 1992.

33. Pruim RHR, Mennes M, van Rooij D, Llera A, Buitelaar JK, Beckmann CF. ICAAROMA: A robust ICA-based strategy for removing motion artifacts from fMRI data. Neuroimage. 2015;112:267-77.

34. Parkes L, Fulcher B, Yücel M, Fornito A. Neurolmage An evaluation of the ef $f$ cacy, reliability, and sensitivity of motion correction strategies for resting-state functional MRI. Neuroimage. 2018;171:415-36.

35. Power JD. A simple but useful way to assess fMRI scan qualities. Neuroimage. 2017;154:150-8

36. Galatzer-Levy IR, Ma S, Statnikov A, Yehuda R, Shalev AY Utilization of machine learning for prediction of post-traumatic stress: A re-examination of cortisol in the prediction and pathways to non-remitting PTSD. Transl Psychiatry 2017; 7. https://doi.org/10.1038/tp.2017.38.

37. Galatzer-Levy IR, Steenkamp MM, Brown AD, Qian M, Inslicht S, Henn-Haase C, et al. Cortisol response to an experimental stress paradigm prospectively predicts long-term distress and resilience trajectories in response to active police service. $J$ Psychiatr Res. 2014;56:36-42.

38. Hastie T. gam: Generalized Additive Models. Hast. T. 2008: R package version 1.0.

39. Hastie T, Tibshirani R. General additive models. Stat Sci. 1986;1:297-318.

40. Jenkinson M, Beckmann CF, Behrens TEJ, Woolrich MW, Smith SM. Review FSL. Neuroimage. 2012;62:782-90.

41. 3.6.1. RDCT. A Language and Environment for Statistical Computing. R Found Stat Comput. 2019.

42. Hervé M RVAideMemoire: Testing and Plotting Procedures for Biostatistics. 2018. https://CRAN.R-project.org/package=RVAideMemoire.

43. Wood SN. Fast stable restricted maximum likelihood and marginal likelihood estimation of semiparametric generalized linear models. J R Stat Soc Ser B Stat Methodol. 2011;73:3-36.

44. Uddin LQ. Salience processing and insular cortical function and dysfunction. Nat Rev Neurosci 2015;16:55-61.

45. Strata P. The emotional cerebellum. Cerebellum 2015;14:570-7.

46. Schutter DJLG, Van Honk J. The cerebellum on the rise in human emotion. Cerebellum. 2005;4:290-4.

47. Timmann D, Drepper J, Frings M, Maschke M, Richter S, Gerwig M et al. The human cerebellum contributes to motor, emotional and cognitive associative learning. A review. Cortex. 2010. https://doi.org/10.1016/j.cortex.2009.06.009. 
48. Holmes SE, Scheinost D, DellaGioia N, Davis MT, Matuskey D, Pietrzak RH, et al. Cerebellar and prefrontal cortical alterations in PTSD: structural and functional evidence. Chronic Stress. 2018;2:247054701878639.

49. Rabellino D, Densmore $M$, Théberge J, McKinnon MC, Lanius RA. The cerebellum after trauma: Resting-state functional connectivity of the cerebellum in posttraumatic stress disorder and its dissociative subtype. Hum Brain Mapp. 2018;39:3354-74.

50. Sussman D, Pang EW, Jetly R, Dunkley BT, Taylor MJ. Neuroanatomical features in soldiers with post-traumatic stress disorder. BMC Neurosci. 2016; 17. https://doi. org/10.1186/s12868-016-0247-x.

51. Thome J, Densmore M, Frewen PA, McKinnon MC, Théberge J, Nicholson AA, et al. Desynchronization of autonomic response and central autonomic network connectivity in posttraumatic stress disorder. Hum Brain Mapp. 2017;38:27-40.

52. Yin Y, Li L, Jin C, Hu X, Duan L, Eyler LT, et al. Abnormal baseline brain activity in posttraumatic stress disorder: A resting-state functional magnetic resonance imaging study. Neurosci Lett. 2011;498:185-9.

53. Ahmadizadeh MJ, Rezaei M. Unilateral right and bilateral dorsolateral prefrontal cortex transcranial magnetic stimulation in treatment post-traumatic stress disorder: A randomized controlled study. Brain Res Bull. 2018. https://doi.org/ 10.1016/j.brainresbull.2018.06.001.

54. Philip NS, Barredo J, van't Wout-Frank M, Tyrka AR, Price LH, Carpenter LL. Network mechanisms of clinical response to transcranial magnetic stimulation in posttraumatic stress disorder and major depressive disorder. Biol Psychiatry. 2018. https://doi.org/10.1016/j.biopsych.2017.07.021.

55. Steudte-Schmiedgen S, Stalder T, Schönfeld S, Wittchen HU, Trautmann S, Alexander $\mathrm{N}$, et al. Hair cortisol concentrations and cortisol stress reactivity predict PTSD symptom increase after trauma exposure during military deployment. Psychoneuroendocrinology. 2015;59:123-33.

56. Lentz L, Silverstone PH, Krameddine Yl. High rates of mental health disorders in civilian employees working in police organizations. Front Psychol. 2020. https:// doi.org/10.3389/fpsyg.2020.01031.

57. Michopoulos V, Norrholm SD, Jovanovic T. Diagnostic biomarkers for posttraumatic stress disorder: promising horizons from translational neuroscience research. Biol Psychiatry. 2015;78:344-53.

\section{ACKNOWLEDGEMENTS}

This work was supported by a $\mathrm{VICl}$ grant (\#453-12-001) from the Netherlands Organization for Scientific Research (NWO) and a consolidator grant from the European Research Council (ERC_CoG-2017_772337) awarded to Karin Roelofs, also supporting WZ, RK, MMH, SBJK, VAvA, and FK. We are particularly grateful to Dutch Police Academy (Politieacademie) for their cooperation. We also thank our former and current colleagues Ingrid Kersten, Naomi de Valk, Geoffrey Bertou, Leonore Bovy, Iris Hulzink, Tiele Dopp, Marijolein Hartgerink, Bart Becker, Madine Zoet, Delphin van Benthem, Job de Brouwer, Lisanne Nuijen, Pepijn van Houten, Klaas van Groesen, Nienke Flipsen, and Joep Trienekens for their help in setting up the study, recruiting participants, and acquiring data, and to Paul Gaalman for his technical assistance in fMRI data acquisition.

\section{AUTHOR CONTRIBUTIONS}

WZ, RK, MMH, SBJK, AM, VAvA, FK, and KR contributed to the study design. WZ, RK, $\mathrm{MMH}$, and SBJK conducted the experiment. WZ performed data analysis. WZ, FK, and KR drafted the paper. WZ, RK, MMH, SBJK, CFB, FK, and KR edited the manuscript. All authors approved the final version of the paper for submission.

\section{COMPETING INTERESTS}

The authors declare no competing interests.

\section{ADDITIONAL INFORMATION}

Supplementary information The online version contains supplementary material available at https://doi.org/10.1038/s41398-022-01798-0.

Correspondence and requests for materials should be addressed to Wei Zhang.

Reprints and permission information is available at http://www.nature.com/ reprints

Publisher's note Springer Nature remains neutral with regard to jurisdictional claims in published maps and institutional affiliations.

(i) Open Access This article is licensed under a Creative Commons adaptation, distribution and reproduction in any medium or format, as long as you give appropriate credit to the original author(s) and the source, provide a link to the Creative Commons license, and indicate if changes were made. The images or other third party material in this article are included in the article's Creative Commons license, unless indicated otherwise in a credit line to the material. If material is not included in the article's Creative Commons license and your intended use is not permitted by statutory regulation or exceeds the permitted use, you will need to obtain permission directly from the copyright holder. To view a copy of this license, visit http://creativecommons. org/licenses/by/4.0/.

(c) The Author(s) 2022 